\title{
Measurement of Chromaticity of Colors Displayed by Switching Two Colors on a Liquid Crystal Display
}

\author{
Chikako Ishizawa $^{\mathrm{a},{ }^{*}, \text { Mozomi Araya }^{\mathrm{b}} \text {, Yoichi Kageyama }}{ }^{\mathrm{a}}$ and Makoto Nishida ${ }^{\mathrm{a}}$ \\ ${ }^{a}$ Graduate School of Engineering and Resource Science, Akita University, Akita 010-8502, Japan \\ ${ }^{\mathrm{b}}$ Department of Computer Science and Engineering, Akita University, Akita 010-8502, Japan \\ *Corresponding Author: ishizawa@ie.akita-u.ac.jp
}

\begin{abstract}
This paper describes the chromaticity measurement of the colors displayed when switching two different colors on a liquid crystal display (LCD). First, the colors displayed on the LCD are measured when red, green, blue, yellow, cyan, and magenta with different gradations are each changed to black. The colors displayed are also measured when changed into colors of the same types but with different gradations. As a result, we confirmed for all cases that the chromaticity of the displayed colors was equal to the average of the two colors that were switched. Next, a method for estimating the chromaticity of the colors displayed through the switching of two colors was proposed. The comparison results between the estimated and measured chromaticity indicate that the proposed method is able to estimate closely the chromaticity of the displayed colors.
\end{abstract}

Keywords: Chromaticity, Liquid crystal display, Sub-pixel, Successive additive color mixing.

\section{Introduction}

Owing to the widespread use of digital and mobile phone cameras, photography has become a ubiquitous activity. However, illegal photography using such devices has generated certain social problems. For example, copyright infringement cases have occurred in which theater screens are captured by a digital camera and the resulting video files are posted onto the Internet ${ }^{(1,2)}$.

To prevent users from taking photos of an image shown on a display or screen, digital watermark methods have been proposed $^{(3-5)}$. However, such methods intend to identify where the image was photographed by detecting specific embedded information (i.e., a watermark) not perceivable by the human eye. Thus, although digital watermarks have the effect of suppressing the use of illegal photographs and image, it is still impossible to prevent the photographs from being taken in the first place.

On the other hand, when two different colors are switched at high speed, it becomes impossible for a human to distinguish them, and the resulting color from the mixing of two original colors can be perceived. This color mixing is called successive additive color mixing ${ }^{(6,7)}$. Displaying such colors on an LCD by using successive additive color mixing may be applied to protect a displayed image. For instance, when images of red and green color are switched, as shown in Fig. 1, a yellow image will be perceived. However, a photograph of the image will show red or green. Thus, the image displayed cannot be captured properly.

It is known that the average color is perceived when applying successive additive color mixing ${ }^{(6,7)}$. However, there are no examples of executing successive additive color mixing on an LCD. In addition, to create an image with swapped colors by modifying the color of the original image, it is necessary to estimate the color displayed when the two colors are switched on the LCD. In this study, we

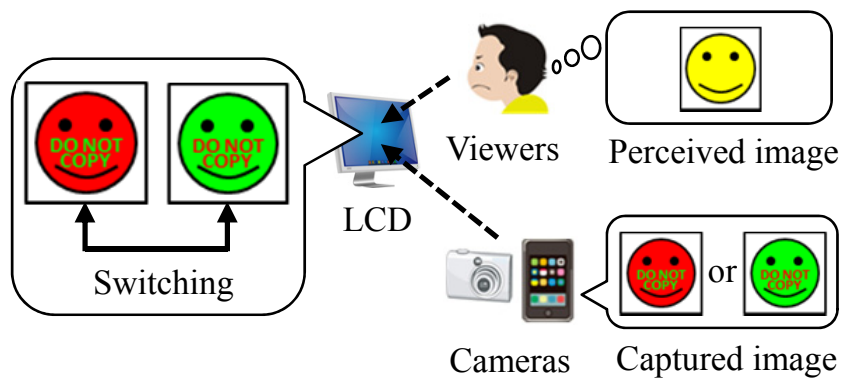

Fig. 1. The copy protection of images achieved by switching two colors. 
measured the chromaticity of the colors displayed when two different colors are switched on an LCD. Furthermore, we propose a method for estimating the chromaticity of the colors displayed through the switching of two colors.

\section{Sub-pixels on the LCD}

Fig. 2 shows the sub-pixels that constitute a single pixel on a LCD. Each sub-pixel outputs a light with the brightness corresponding to the RGB levels. A color is displayed through a mixture of three outputted lights ${ }^{(8)}$.

When the colors are switched, each sub-pixel takes one of the four following states:

- State 1: the lights are constantly outputted.

- State 2: the lights are turned on and off repeatedly.

- State 3: the strengths of the lights are changed repeatedly.

- State 4: the lights are not outputted.

Fig. 3 shows an example of the above states.

Usually, the switching between lights with low and high levels of brightness, such as in state 2 , is quick on an LCD. However, the switching of lights with a mid-level of brightness, such as in state 3 , is slow. Therefore, it is

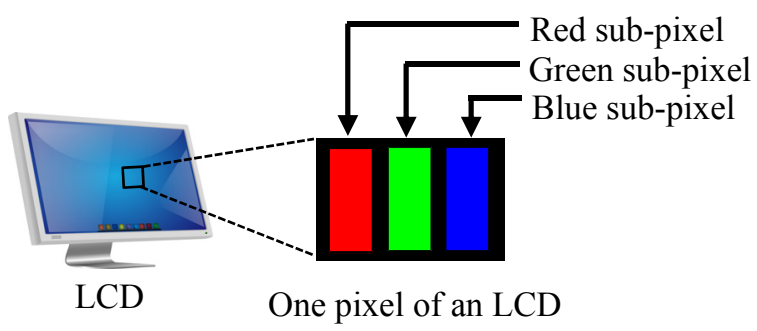

Fig. 2. Sub-pixels of an LCD.

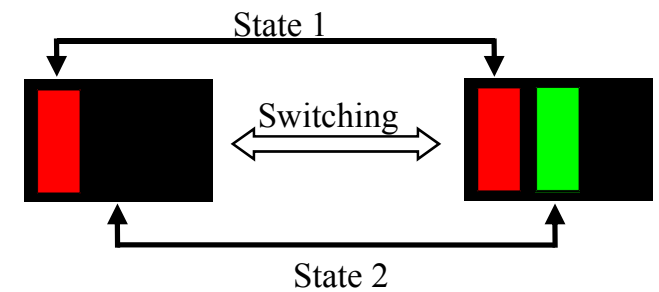

(a) Switching of red (left) and yellow (right).

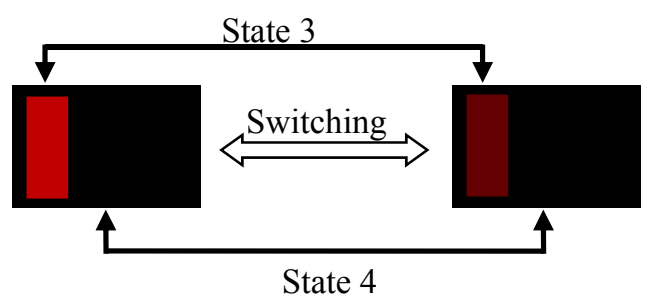

(b) Switching of mid-level brightness reds.

Fig. 3. States of sub-pixels when switching colors. necessary to check whether a color displayed during state 2 or 3 is equal to the average of the two switching colors.

\section{Chromaticity of the displayed color}

\subsection{Measurement method}

(1) Outline of the measurement

Fig. 4 shows a schematic of the experimental conditions The color on the LCD screen was measured using a colorimeter. The colorimeter was put to contact with the screen, and the circumference of the light receiving window was covered by a black visor of the opacity to block any environmental light. Therefore only lights output from the LCD were measured. The specifications of the LCD (EIZO CG275W) and the colorimeter (KONICA MINOLTA CL-200) used in the experiment are listed in Tables 1 and 2.

The switched colors were alternately displayed at a speed of 61 fps based on previous synchronization results and the refresh rate of the LCD. We previous examined the relationship between the switching speed and flicker using LCDs with a refresh rate of $60 \mathrm{~Hz}^{(9-11)}$. Our experiments clarified that the flicker is reduced when using a switching speed of $61 \mathrm{fps}$. A time lag occurs from when the command is given until the process of changing the color is executed. Therefore, it is necessary to use a switching speed that is slightly faster than the refresh rate for synchronization with this rate. The integral time of the colorimeter used is 100 ms. The mean of the integral time is provided as the measurement result. Therefore, we can obtain the chromaticity of the colors displayed from the switching. The chromaticity was obtained as XYZ values.

(2) Colors used

Table 3 lists the details of the colors used for the switching in state 2 . Red, green, and blue are the primary

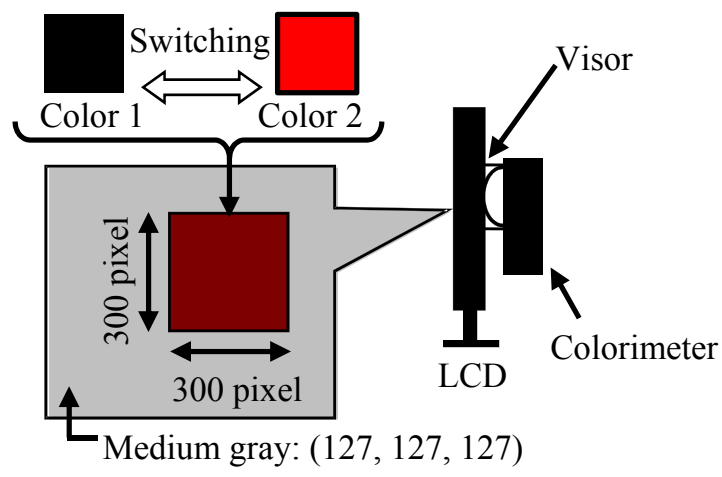

Fig. 4. Schematic of the experimental conditions. 
Table 1. Specifications of the LCD.

\begin{tabular}{|l|l|}
\hline Size & 27.0 in. \\
\hline Resolution & 2560 dots $\times 1440$ lines \\
\hline Number of colors & True color $(16,777,216$ colors $)$ \\
\hline Response speed & $\begin{array}{l}12 \mathrm{~ms} \text { (black }- \text { white }- \text { black }) \\
6 \mathrm{~ms} \text { (half tone value) }\end{array}$ \\
\hline Refresh rate & $60 \mathrm{~Hz}$ \\
\hline $\begin{array}{l}\text { Color temperature of } \\
\text { reference white }\end{array}$ & $6498 \mathrm{~K}$ \\
\hline $\begin{array}{l}\text { Chromaticity } \\
\text { coordinate of color } \\
\text { gamut }\end{array}$ & $\begin{array}{l}\text { green }(0.6798,0.03090) \\
\text { blue }(0.1493,0.0494)\end{array}$ \\
\hline Gamma value & 2.20 \\
\hline
\end{tabular}

Table 2. Specifications of the colorimeter.

\begin{tabular}{|l|l|}
\hline Receptor & Silicon photocell \\
\hline $\begin{array}{l}\text { Relative spectral } \\
\text { response }\end{array}$ & $\begin{array}{l}\text { Closely matches CIE standard } \\
\text { observer curves } \mathrm{x}(\lambda), \mathrm{y}(\lambda) \text {, and } z(\lambda) \\
\text { Within 6\% (f1') of the CIE spectral } \\
\text { luminous efficiency V( } \lambda)\end{array}$ \\
\hline Measuring range & $\begin{array}{l}0.1 \text { to 99,990 lx (Chromaticity:5 lx } \\
\text { or above) in four automatically } \\
\text { selected ranges }\end{array}$ \\
\hline Response time & 0.5 sec. (continuous measurement) \\
\hline
\end{tabular}

Table 3. Gradation values (RGB values) of the colors used for switching in state 2.

\begin{tabular}{|c|c|c|c|c|c|c|}
\hline $\begin{array}{c}\text { Gradation } \\
\text { value }\end{array}$ & Red & Green & Blue & Yellow & Cyan & Magenta \\
\hline 0 & $(0,0,0)$ & $(\mathbf{0}, \mathbf{0}, \mathbf{0})$ & $(0,0,0)$ & $(0,0,0)$ & $(0,0,0)$ & $(\mathbf{0}, \mathbf{0}, \mathbf{0})$ \\
\hline 31 & $(31,0,0)$ & $(0,31,0)$ & $(0,0,31)$ & $(31,31,0)$ & $(\mathbf{0}, 31,31)$ & $(31,0,31)$ \\
\hline 63 & $(63,0,0)$ & $(0,63,0)$ & $(0,0,63)$ & $(63,63,0)$ & $(0,63,63)$ & $(63,0,63)$ \\
\hline 95 & $(95,0,0)$ & $(0,95,0)$ & $(0,0,95)$ & $(95,95,0)$ & $(0,95,95)$ & $(95,0,95)$ \\
\hline 127 & $(127,0,0)$ & $(0,127,0)$ & $(0,0,127)$ & $(127,127,0)$ & $(0,127,127)$ & $(127,0,127)$ \\
\hline 159 & $(159,0,0)$ & $(0,159,0)$ & $(0,0,159)$ & $(159,159,0)$ & $(0,159,159)$ & $(159,0,159)$ \\
\hline 191 & $(191,0,0)$ & $(0,191,0)$ & $(0,0,191)$ & $(191,191,0)$ & $(0,191,191)$ & $(191,0,191)$ \\
\hline 223 & $(223,0,0)$ & $(0,223,0)$ & $(0,0,223)$ & $(223,223,0)$ & $(0,223,223)$ & $(223,0,223)$ \\
\hline 255 & $(255,0,0)$ & $(0,255,0)$ & $(0,0,255)$ & $(255,255,0)$ & $(0,255,255)$ & $(255,0,255)$ \\
\hline
\end{tabular}

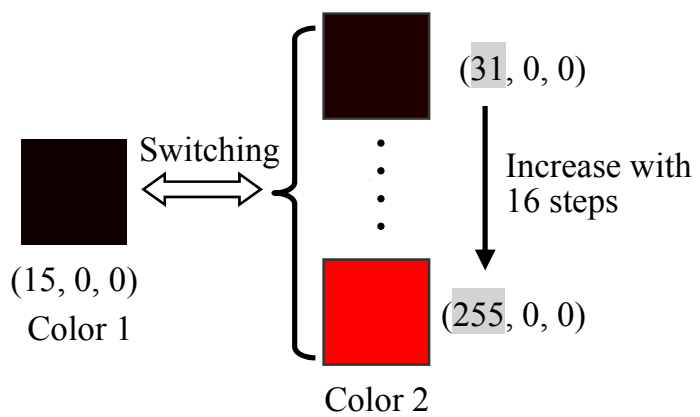

Fig. 5. Combinations of colors used for switching in state 3 .

colors of the RGB colorimetric system, and yellow, cyan, and magenta are the complementary colors. The gradation values (RGB values) of these colors are changed in increments of 32 steps within the range of 0 to 255 . A total of 54 colors were switched ( 9 gradations $\times 6$ color types). The color displayed was measured when each color was changed to black, as shown in Table 3. The measurements were repeated ten times for each color, and the average values were used.

The colors used for the switching in state 3 were red, green, and blue, which were changed in increments of 16 steps, with gradation values ranging from 15 to 255 . Fig. 5 shows a combination method for color switching. Two colors of the same type but a different gradation were selected. For example, red with a gradation value of 15 was combined with red with the gradation value other than 15 . A total of 360 sets were combined ( 120 sets $\times 3$ color types).

To be considered, the chromaticity of each color used for switching during states 2 and 3 was also measured.

\subsection{Measurement results and discussion}

(1) Chromaticity of the color displayed by repeatedly turning the lights on and off (state 2)

Fig. 6 shows an example of the measurement results. The horizontal axis expresses the gradation level (RGB values) of the color, and the vertical axis expresses the chromaticity (XYZ values). In Fig. 6, a cross expresses the chromaticity of the displayed color, and a circle expresses the chromaticity of the color used for the switching. The chromaticity increases in a curve with an increase in the gradation. Table 4 shows the XYZ values of red, green, and 
blue with a gradation value of 255 . The comparison results using the maximum values show that the XYZ values of the colors displayed are half those of the switching colors. It is thought that the light quantum from the sub-pixels is halved because black is used for the switching. A similar result was

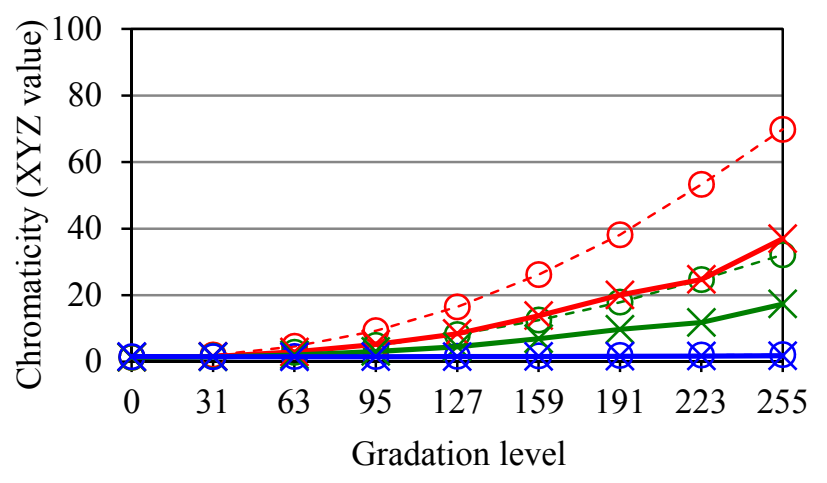

(a) Red

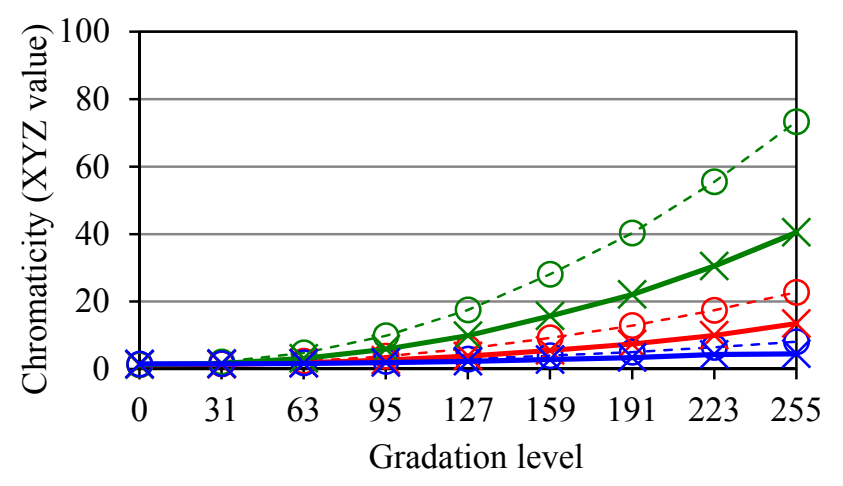

(b) Green

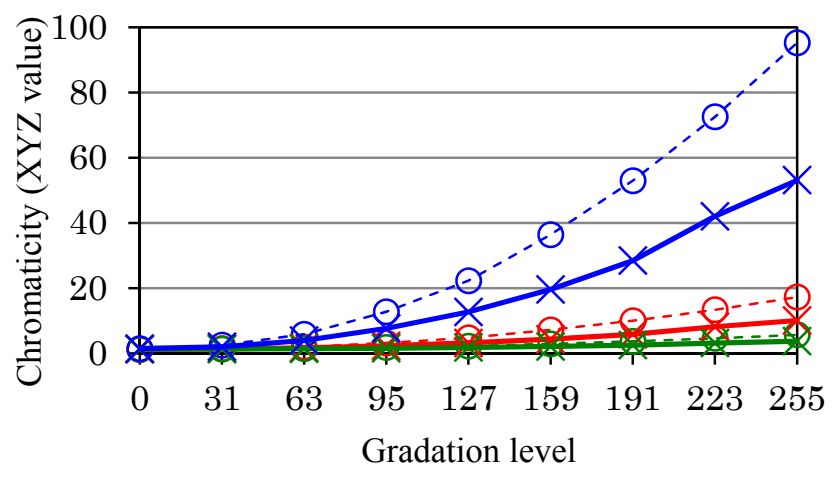

(c) Blue

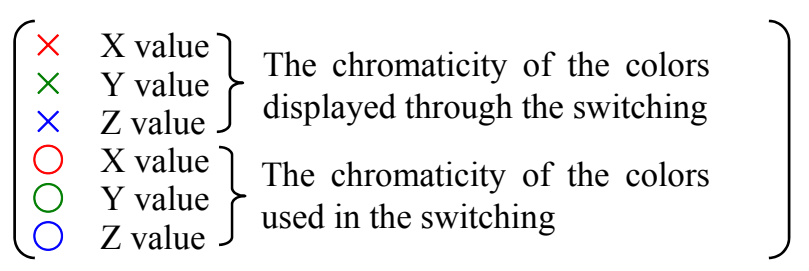

Fig. 6. The measurement results in state 2 . shown for yellow, cyan, and magenta. This result suggests that the chromaticity of the color displayed through repetitively turning on and off the lights is calculated based on the average colors used in the switching.

When yellow and black are switched, the red and green sub-pixel lights are turned on and off repeatedly. The chromaticity of the color displayed by switching between yellow and black was compared with the chromaticity of the color displayed by switching between red and green. Similarly, the switching of cyan was compared with the switching of green and blue, and the switching of magenta was compared with the switching of red and blue. Fig. 7 shows the results for yellow. The yellow marks in Fig. 7 indicate the chromaticity of the color displayed through the switching between yellow and black. The black marks in Fig. 7 indicate the total of the chromaticity obtained through the switching of red and the switching of green. The chromaticity of the color displayed through the switching between yellow and black is equal to the total chromaticity of switching between red and green. A similar result was obtained between cyan and magenta. This result suggests that the calculation of the chromaticity is possible using the method of additive color mixing when the lights of the sub-pixels are turned on and off repeatedly.

(2) Chromaticity of color displayed through a repeated change in the strength of the lights (state 3 )

The chromaticity of the displayed colors was compared with the average of the two colors used in the switching. Fig. 8 shows the chromaticity of the color displayed when red with a gradation level of 127 is combined and switched with red with a gradation level of 15 to 255 . It was confirmed that the chromaticity of the displayed color nearly match the average chromaticity of the two switching colors. Similar results were also shown through combinations of green and blue using other gradation. This

Table 4. Chromaticity of gradation 255 .

\begin{tabular}{|c|c|c|c|}
\cline { 3 - 4 } \multicolumn{1}{c|}{} & Displayed color & Switched color \\
\hline \multirow{4}{*}{ Red } & X value & 69.8158 & 36.9339 \\
\cline { 2 - 4 } & Y value & 32.1200 & 17.3300 \\
\cline { 2 - 4 } & Z value & 2.0899 & 1.7568 \\
\hline \multirow{4}{*}{ Blue } & X value & 22.7204 & 13.4674 \\
\cline { 2 - 4 } & Y value & 73.3100 & 40.4900 \\
\cline { 2 - 4 } & Z value & 8.0913 & 4.4773 \\
\hline \multirow{4}{*}{} & X value & 17.2438 & 10.0535 \\
\cline { 2 - 4 } & Y value & 5.6900 & 3.6900 \\
\cline { 2 - 4 } & Z value & 95.2877 & 53.1285 \\
\hline
\end{tabular}




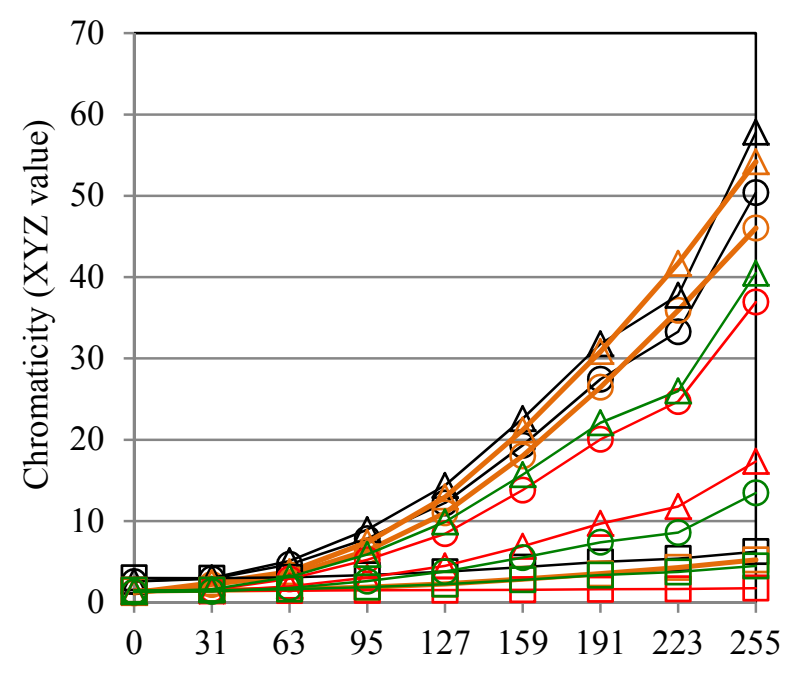

Gradation level

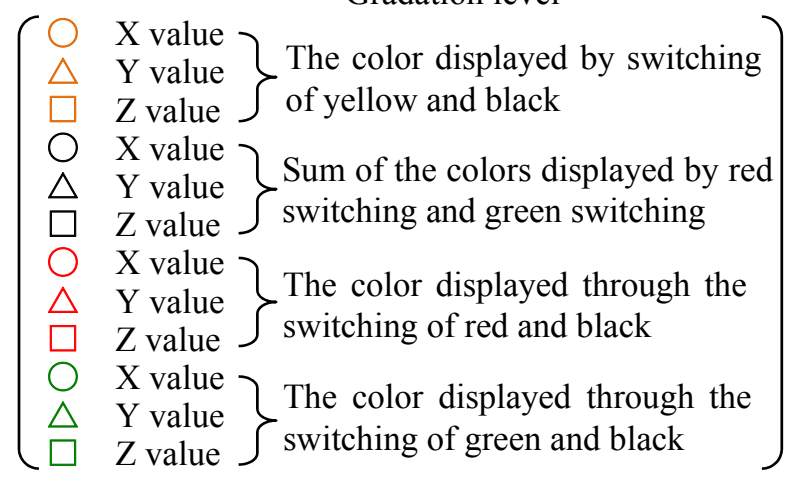

Fig. 7. The measured and totaled chromaticity.

result also suggests that the chromaticity of the color displayed by repeated changes in the strength of the lights is based on the average chromaticity of the colors used in the switching.

\section{Estimation of chromaticity}

\subsection{Proposed method}

The proposed method consists of three steps. Usually, the chromaticity of a color displayed on an LCD is expressed using the XYZ color system. In the proposed method, the chromaticity is also calculated using the XYZ values.

- Step 1: Calculate the maximum chromaticity of the sub-pixels

The maximum chromaticity of each sub-pixel is calculated to define the characteristics of the gradation curve. Three LCD settings and the $\mathrm{Y}$ value obtained through the measurements are used to calculate the maximum chromaticity. The calculation procedure is as follows:

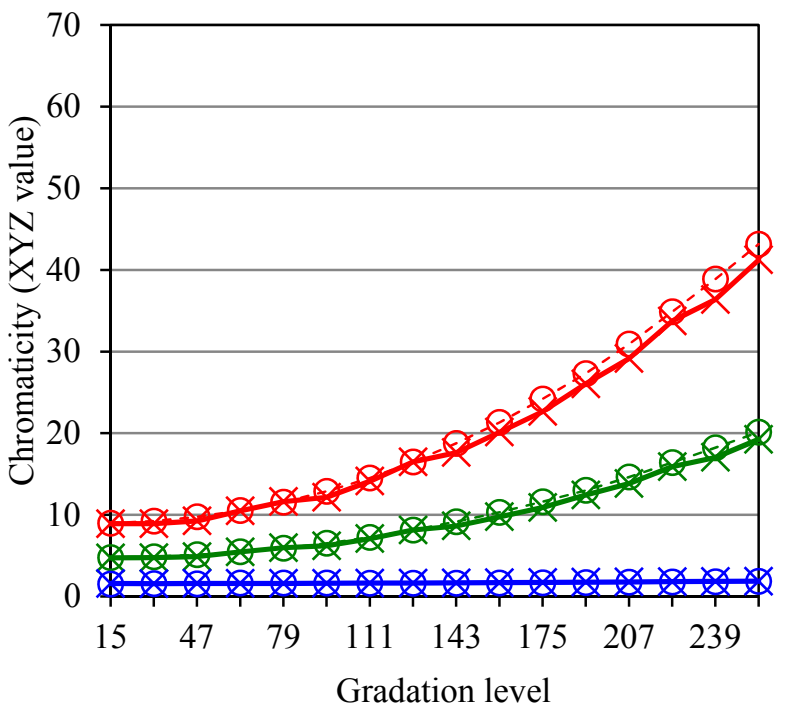

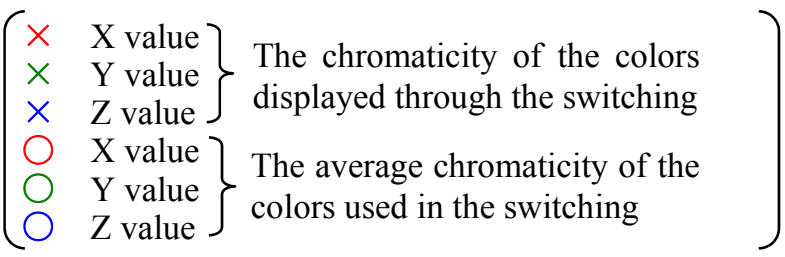

Fig. 8. The measurement results in state 3 (the switching with the red of gradation level 127).

(i) Convert the color temperature of the reference white of the LCD settings into a chromaticity coordinate $\left(x_{\mathrm{w}}\right.$, $\left.y_{\mathrm{w}}\right)$ on an $x y$ chromaticity diagram.

(ii) Calculate the $X Y Z$ values $\left(X_{w}, Y_{w}, Z_{w}\right)$ of the reference white by applying the ratio of $X, Y$, and $Z$ to the maximum luminance. This calculation is as follows:

$$
\left.\begin{array}{l}
\mathrm{X}_{\mathrm{w}}=\mathrm{L} \times x_{\mathrm{w}} / y_{\mathrm{w}} \\
\mathrm{Y}_{\mathrm{w}}=\mathrm{L} \times 1 \\
\mathrm{Z}_{\mathrm{w}}=\mathrm{L} \times\left(1-x_{\mathrm{w}}-y_{\mathrm{w}}\right) / y_{\mathrm{w}}
\end{array}\right\}
$$

where $x_{\mathrm{w}}$ and $y_{\mathrm{w}}$ are the chromaticity coordinates of the reference white, and $\mathrm{L}$ is the maximum luminance value of the displayed colors, which is obtained by summing each Y value of red, green, and blue with a gradation of 255 .

(iii) Calculate the maximum XYZ value of each sub-pixel by applying the ratio of red, green, blue to the reference white, the calculation of which is as follows:

$$
\begin{aligned}
& \mathrm{X}_{\mathrm{R}}=\mathrm{X}_{\mathrm{w}} \times x_{\mathrm{R}} /\left(x_{\mathrm{R}}+x_{\mathrm{G}}+x_{\mathrm{B}}\right) \\
& \mathrm{Y}_{\mathrm{R}}=\mathrm{Y}_{\mathrm{w}} \times y_{\mathrm{R}} /\left(y_{\mathrm{R}}+y_{\mathrm{G}}+y_{\mathrm{B}}\right) \\
& \mathrm{Z}_{\mathrm{R}}=\mathrm{Z}_{\mathrm{w}} \times z_{\mathrm{R}} /\left(z_{\mathrm{R}}+z_{\mathrm{G}}+z_{\mathrm{B}}\right)
\end{aligned}
$$

where $x_{\mathrm{R}}, y_{\mathrm{R}}$, and $z_{\mathrm{R}}$ are the chromaticity coordinates of the brightest red within the color gamut of the LCD; $x_{\mathrm{G}}, y_{\mathrm{G}}$, and $z_{\mathrm{G}}$ are the chromaticity coordinates of the brightest green; and $x_{\mathrm{B}}, y_{\mathrm{B}}$, and $z_{\mathrm{B}}$ are the chromaticity coordinates of the brightest blue. The maximum XYZ value of the red sub-pixel is obtained through expression (2). The expressions of green and 
blue are also defined in this same way.

- Step 2: Define the characteristic of the gradation curve of the sub-pixels

Define the characteristic of the gradation curve depending on the state of the sub-pixels. Expression (3) is used when the sub-pixels output a constant light (state 1). Expression (4) is used when the sub-pixels are blinking (states 2 and 3 ).

$$
\begin{aligned}
& \mathrm{X}=\mathrm{X}_{\mathrm{R}} \times(\mathrm{N} / 255)^{\gamma} \\
& \mathrm{Y}=\mathrm{Y}_{\mathrm{R}} \times(\mathrm{N} / 255)^{\gamma} \\
& \mathrm{Z}=\mathrm{Z}_{\mathrm{R}} \times(\mathrm{N} / 255)^{\gamma} \\
& \mathrm{X}=\mathrm{X}_{\mathrm{R}} / 2 \times(\mathrm{N} / 255)^{\gamma} \\
& \mathrm{Y}=\mathrm{Y}_{\mathrm{R}} / 2 \times(\mathrm{N} / 255)^{\gamma} \\
& \mathrm{Z}=\mathrm{Z}_{\mathrm{R}} / 2 \times(\mathrm{N} / 255)^{\gamma}
\end{aligned}
$$

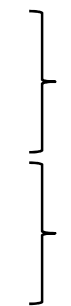

where $\mathrm{N}$ is the gradation value of the sub-pixels, and $\gamma$ is the gamma value of the LCD.

- Step 3: Estimate the chromaticity of the displayed colors The chromaticity of a displayed color is calculated based on the total chromaticity of each sub-pixel. For instance, when red and green are switched, the chromaticity of yellow is determined to be the sum of the chromaticity of the red and green sub-pixels as represented through expression (4). In addition, when red and yellow are switched, the chromaticity of the displayed color is determined to be the sum of the chromaticity of the red sub-pixels as represented through expression (3), and the chromaticity of the green sub-pixels as represented through expression (4).

\subsection{Experimental results and discussion}

To examine the usefulness of the proposed method, the estimated chromaticity was compared with the measured values provided in chapter 3 . We confirmed whether the proposed method could estimate the chromaticity of the color shown in Table 3 or not. Fig. 9 shows the comparison results when the red shown in Table 3 was estimated. In Fig. 9, where the horizontal axis expresses the gradation level (RGB values) of the color, and the vertical axis expresses the chromaticity (XYZ values). The results show that the estimated chromaticity of a color displayed through the switching of two colors almost matches the measured chromaticity. Therefore, the chromaticity of the color displayed through the switching of colors shown in table 3 will be supposed with half values of the chromaticity shown in Fig. 9. This result suggests that the proposed method is able to estimate closely the chromaticity of the displayed colors through the switching of various colors.
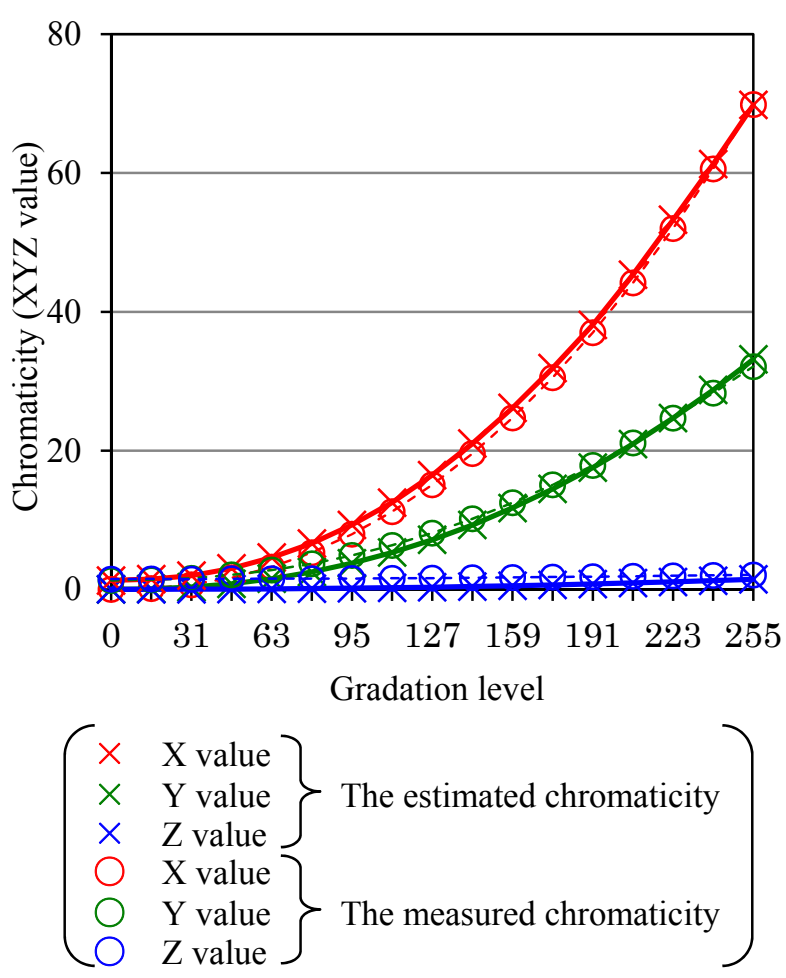

Fig. 9. The estimated and measured chromaticity (when the red shown in Table 3 was estimated).

\section{Conclusions}

This paper measured the chromaticity of the colors displayed when two different colors are switched on an LCD. Additionally, a method for estimating the chromaticity of the colors displayed through the switching of two colors was proposed. The following results were obtained:

(1) The chromaticity of the colors displayed through the switching of two colors was equal to the average chromaticity of the two colors used in the switching.

(2) The proposed method was able to estimate the chromaticity of the colors displayed when switching two colors on an LCD.

For our future study, we intend to propose a method for choosing two colors to switch based on the desired color of the original image.

\section{Acknowledgment}

The authors would like to thank Dr. T. Takahashi, Akita University for his help in conducting these experiments. This work was supported by JSPS KAKENHI, Grant Number 15K00145. 


\section{References}

(1) Nikkei Win PC Website, http://pc.nikkeibp.co.jp/article/news/20090817/101786 $7 /$

(2) CNET News Website, http://www.cnet.com/news/google-glass-at-the-moviesgets-man-interrogated/

(3) H. Nakamura, S. Gohshi, R. Fujii, H. Ito, M. Suzuki, S. Takai, and Y. Tani : "A Digital Watermark that Survives after Re-shooting the Images Displayed on a CRT Screen", Journal of the Institute of Image Information and Television Engineers, Vol. 60, No. 11, pp. 1778-1788, 2006

(4) H. Yamada, R. Fujii, M. Suzuki, H. Ito, S. Takai, Y. Tani, S. Gohshi, K. Majima, and H. Nakamura: "A Watermark System that Survives after Re-shooting a Screen", Proceedings of the 2008 IEICE General Conference, p. 129, 2008

(5) M. Suzuki, H. Yamada, K. Asai, T. Murakami, K. Majima, M. Abe, and M. Kawamata: "Method to Specify Time and Space Axis of Digital Watermark against Re-shot Image", Proceedings of 8th Forum on Information Technology, pp. 307-308, 2009

(6) The Color Science Association of Japan: "Color Science Handbook [New edition]", University of Tokyo Press, 1998

(7) Vision Society of Japan: "Visual Information Processing Handbook", Asakura Publishing Co., Ltd., 2000

(8) I. Ohishi, T. Hatada, and T. Tamura: "Basis of the display", Kyoritsu Shuppan Co., Ltd., 2001

(9) C. Ishizawa, S. Takaki, A. Katsuhara, M. Suzuki, Y. Kageyama, and M. Nishida: "Influence of Luminance Change in Subpixels on the Occurrence of Flicker Investigated by Alternating Display of Colors on a Liquid Crystal Display", International Journal of the Society of Materials Engineering for Resources, Vol. 20, No. 1, pp. 97-102, 2014

(10)C. Ishizawa, R. Sato, S. Takaki, and M. Nishida: "Estimation of Colors Perceived by Alternating Display of Colors on a Liquid Crystal Display", The Seventh Inter. Conf. on Mater. Eng. for Resources, pp. 291-296, 2013

(11)C. Ishizawa, R. Sato, Y. Kageyama, and M. Nishida: "Method for Estimating Chromaticity of Colors Displayed by Successive Additive Color Mixing on a Liquid Crystal Display", The SICE Annual Conference 2014, pp. 1976-1979, 2014 